\title{
Study of Combined Action of Papaya Leaf Extract, Pomegranate and Lemon - A Review
}

\author{
Dr.Bandyopadhyay ${ }^{1} \mid$ Prithweejit Bhattacharyya $^{2} \mid$ Lopamudra Banerjee ${ }^{2} \mid$ Pallabi $^{\text {Pal }}{ }^{2} \mid$ Shatarghya \\ Das Mazumder ${ }^{2}$
}

${ }^{1}$ H.O.D and Associate Professor, Department of Food technology, Guru Nanak Institute of Technology, Kolkata, India. 2UG Students, Department of Food technology, Guru Nanak Institute of Technology, Kolkata, India.

To Cite this Article

Dr.Bandyopadhyay, Prithweejit Bhattacharyya, Lopamudra Banerjee, Pallabi Pal and Shatarghya Das Mazumder, "Study of Combined Action of Papaya Leaf Extract, Pomegranate and Lemon - A Review", International Journal for Modern Trends in Science and Technology, Vol. 06, Issue 05, May 2020, pp.: 67-71; https://doi.org/10.46501/IJMTST060511

\section{Article Info}

Received on 24-March-2020, Revised on 24-April-2020, Accepted on 28-April-2020, Published on 02-May-2020.

\section{ABSTRACT}

The main motive of this review study is to focus on the essential components present in papaya leaves, pomegranate juice and sweet lime which is mausambi juice and a result of their combined effect on human health with enhanced beneficial components such as saponins, polyphenols etc. The leaves of papaya are generally considered as waste but their extracts have various health benefits. It contains calcium, magnesium, iron, and vitamins $A, C, E, K$ and $B$. It is said that Saponin which is a component present in papaya leaves have anticancer capacity as well as reduces excessive oxidative stress. Here, papaya leaf extract can be added to pomegranate lemonade where both these fruits have predominant nutritional value. Pomegranate boosts immunity and aids in digestion. It also has anti-inflammatory properties. It is loaded with Vitamin-C and is also antioxidant-rich. Lemon on the other hand also contains a lot of minerals and vitamins, it also reduces oxidative stress. As a result it is an approach of a health drink having medicinal values. The positive effect of all the above extracts when combined can produce immense health benefits in the human body and can act as suitable nutraceutical with chances of less side effects and allergic symptoms.

KEYWORDS: Anti-inflammatory, antioxidant, immunity, nutraceutical, saponin.

Copyright (C) 2014-2020 International Journal for Modern Trends in Science and Technology DOI: https://doi.org/10.46501/IJMTST060511

\section{INTRODUCTION}

The main aim of our study is to use Carica papaya leaf extract as a base to utilize its medicinal value and fortify it with commercially available pomegranate lemonade. papaya leaf extract has anti-cancer properties, added to that it also has a lot of health benefits such as- it may aid in digestion as it contains fiber, it has antioxidant properties, it may lower blood sugar level and may increase blood platelets count in dengue affected patients. Therefore, being cost effective, papaya leaf extract may solve a lot of health problems.
Carica papaya is usually cultivated in tropical regions, it's commonly called as papaya in this part of the world and it's from the family "Caricaceae". Papaya grows best in a well-drained, well aerated and rich organic matter soil, $\mathrm{pH} 5.5$ - 6.7 (Priyanka et al. [1]), while water logging of soils often results in the death of tress within 3-4 days (Storey [2]).The leaves also contain active components such as papin, chymopapin, cystain, ascorbic acid, flavonoids, cyanogenic glucosides that increases the total antioxidant power in blood and reduce lipid preoxidation level. Amount of total 
phenolics, total flavonoids and antioxidant capacity of fruits were more than that of vegetables. American daily intake of phenolics, flavonoids and antioxidants from fruits and vegetables was estimated to be $450 \mathrm{mg}$ GAE, $103 \mathrm{mg}$ catechin equivalents and $591 \mathrm{mg} \mathrm{VCE}$, respectively as showed by the works of (Ock Kyoung et al. [3]).The leaves have been researched for its medicinal uses and have been documented in literature for its use by natives of various parts of the world for its anti- inflammatory, antitumor, anti -diabetic effects among others. Recently the haemostatic property and beneficial effects of Carica papaya leaves in curing the dengue infected patients has been reported. Reports on different parts of C. papaya have been published, but still a comparative study is to be needed to study the difference in medicinal values of different parts of this plant growing in normal conditions and the C.papaya growing in semi-arid region like Kachchh region. As per the findings of (Nor Arzina Norahmad et al. [4]), which was done to understand the flavonoids component present in papaya leaves by High Performance Liquid chromatography Technique (HPLC) method by using freeze dried papaya leaves where the mobile phase consisted of two solvents mainly, $0.01 \%$ Trifluroacetic acid (TFA as Solvent A) and acetonitrile having $0.01 \%$ TFA (as Solvent B) and Waters Symmetry C18 Column, 300Å, $5 \mu \mathrm{m}$, $3.9 \mathrm{~mm} \times 150 \mathrm{mmin}$ in Milford, USA was used as the stationary phase. Carica papaya, locally known as papaya pear or "pawpaw", originates from the Caricaceae family. The ability of the leaves to be used as a cure for these diseases is most likely due to the presence of bioactive com-pounds and multiple phytochemicals in the leaves. Carica papaya leaf extracts have hindered its potential due to its bitterness, greatly believed to be caused by saponins, greatly believed to be caused by saponins. Conventional methods for the extraction of saponins are usually maceration (soaking) and the Soxhlet method in reflux. Here the alkaloids, polyphenol, tannins and saponins are estimated to analyze them.

\section{Papaya leaf Benefits}

Carica papaya is a fast growing plant with height reaching $3-10 \mathrm{~m}$. Papaya leaves are $70 \mathrm{~cm}$ long. Carica papaya is one of the accepted species in the genus Carica of the family Caricaceae. According to the findings of (Susmita Sengupta et al. [5]), papaya leaves are rich in protease and amylase.
These two enzymes help in breaking protein, carbohydrate and minerals and finally aids in digestion. As per studies of (Zuhrotun Nisa F et al. [6]), leaf extracts of papaya plant has the potential to act as anticancer agent by undergoing the mechanism of anti-proliferation and apoptosis induction. It also has immunomodulatory properties. It is said that during dengue fever, the platelet count lowers and that is a major concern to somehow keep the count in proper number. Several researchers have tested the possible ways to cure this issue in dengue affected persons and finally they found some essential components in papaya leaf extract that will serve the purpose. Papaya leaf consists of compounds like flavonoids and alkaloids as carpaine, anthraquinone, and saponin. Papaya leaf has good antioxidant property as well as free radical scavenging property. It also may stabilize RBC membrane and control hemolysis. Papaya leaf extract is capable increasing blood platelet count because it helps in increasing ALOX12 (arachidonate 1-lipoxygenaseor platelet type lipoxygenase) by 15 times. This enzyme helps in increasing blood platelet count by stimulating megakaryocytes. Serine protease NS2B and NS3 these are vital in dengue virus replication, but their action gets obstructed by quercetin, one of the flavonoids in papaya leaf. Some studies also give us the information that papaya leaf extract cleanses our blood by lowering the cholesterol levels. Many dengue affected patients have been reported to be suffering from severe neurological problems as showed by the works of (Rajesh Verma et al. [7]), although the work which was done by (Saurabh Pandey et al. [8]) showed the beneficial role of papaya leaves on the central nervous system showing that it can be helpful to keep nervous disorders away from dengue affected patients. As per the work of (Vuong et al. [9]) and (Amran et al. [10]), saponin is the main component responsible for the bitterness of papaya leaves. The bitter aftertaste in papaya leaf extracted juice is due to the presence of saponin compound making them non-consumer friendly. Saponins are structurally amphiphilic, containing hydrophilic (carbohydrate) and hydrophobic (steroids or terpenes). Other components in papaya leaf include tannin, cardiac glycosides, alkaloids etc. 


\section{Pomegranate Benefits}

Pomegranate (Punica granatum L.) belongs to the Punicacea family. It is one of the important and commercial horticultural fruits which are generally very well adapted to the Mediterranean climate. Different parts of its tree such as leaves, fruits and bark skin have been used traditionally for their medicinal properties and for other purposes such as in tanning as reported by (Kulkarni et. al [11]). Pomegranate juice having a dark rich color shows the presence of high antioxidant content mainly anthocyanins as reported by the works of (Sing R.P

et al. [12]). It is proved to have high antioxidant activity. Pomegranate also has anti-inflammatory properties that may include heart disease; obesity Pomegranate also may lower blood sugar level if consumed on a regular basis. Individuals consuming pomegranate juice have reported to gain an increase in the red blood cell count and hemoglobin concentration as reported by the works of (Eirini Manthou et al. [13]). It is a source to fight arthritis by blocking the enzymes that damage the joints. It may also lower the risk of heart disease. The main fatty acid of pomegranate is punicic acid that helps to protect against heart disease. It lowers the triglyceride level and improves the triglyceride-HDL ratio.

\section{BENEFicial ROLES OF LEMON}

The high content of Vitamin C in "Mausambi" or sweet lime helps in alleviating inflammation and swelling and hence plays a pivotal role in treating symptoms of osteoarthritis and rheumatoid arthritis. It also enhances calcium absorption, stimulates the cell to build bone and promote overall bone health. Lemon also has antioxidant properties in it. Due to the presence of vitamin C content, it also prevents asthma. Lemon also increases the iron absorption ability of the body and boosts up the immunity of the body. Thus lemon does have some significant nutritional benefits. As per the works which were done by (Chun et al. [14]) and (Xu et al.[15]) it showed that citrus fruits have the highest antioxidant activity due to the presence of ample amount of flavonoids, Vitamin C and carotenoids. Due to the presence of platelet inhibitory affect, citrus lemons are reported to show usefulness by acting as an antidote against certain venoms according to the work done by (Arias et al. [16]).

\section{Discussions}

Our topic is to study about the combined action of papaya leaf extract, pomegranate and lemon juice to develop a health drink. Here, we have selected pomegranate and lemon juice to carry out further experiment. Because of the individual nutritional benefits that each of these three hold may come out well as a productive health drink that can be a solution to a lot of health problems. We searched for few experiments that are well established so that this concept can be carried out further. Many studies have already proved that individually these three products have good amount of antioxidants. Especially papaya leaf extracts are proven to increase the platelet count in patients suffering from dengue as showed by the works of (B.K Srikanth et al. [17]). Our aim was to not only develop a health drink but also to make it appealing as much as it could be keeping their nutritional aspects intact. Papaya leaf, pomegranate and lemon have their individual nutritional benefits which can extracted and utilized for human health betterment purpose and this study in an approach to develop a health drink, combining these three in correct proportions keeping their chemical nature in mind. As per the findings which were published by the Department of Medicine from University of Peradeniya (Senanayake AM Kularatne [18]), Sri Lanka and (Hettige S. [19]), it was seen that the bitterness was reduced by addition of sugar powder; but sugar addition creates an obstruction for the medicine to be applicable for diabetic patients as well. The experiments which got carried out by the researchers gave this study a validation which was needed for further queries and to take the concept forward.

\section{Data Analysis}

According to the study of (Thao T. Nguyen et al. [20]) chromatography of papaya leaf extract is as

follows: -

The above figure shows the chromatography of Carica papaya leaf extracts as follows,

A. Base Peak Chromatograms (BPC) of leaf juice, brewed leaf juice, leaf decoction in positive mode and $\mathrm{B}$. In negative mode;

C. BPCs from the three replicates of leaf juice samples in positive mode

D. In negative mode. 


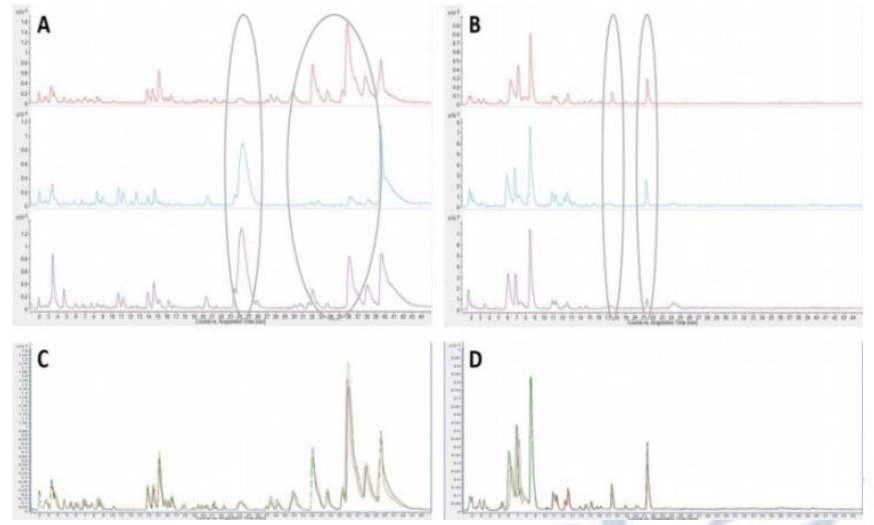

The chromatographic region between 2 to $45 \mathrm{~min}$ is shown as it contained almost all of the chromatographic peaks.

According to (R. Fatin Najwa and Azrina Azwal, [21]) the citric acid content of lemon juice is found to be in the range 42.5 to $50 \mathrm{mg}$. According to (Sadiye Gozlekci et al. [22]), the total phenolic content of pomegranate juice ranged from 784.4 to $1551.5 \mathrm{mg}$ GAE/L (gallic acid equivalent/liter). Optimal extraction condition is $70^{\circ} \mathrm{C}$ for 20 minutes. With a water to leaf ratio $100: 7.5 \mathrm{mg} / \mathrm{L}$. Higher level of polyphenol is obtained keeping water as a solvent rather than organic solvents. $1 \mathrm{~kg}$ crude dried papaya leaf contains $6.3 \%$ of polyphenols.

\section{Conclusion}

This study gave us a lot of information regarding papaya, papaya leaf, pomegranate and lemon. Some of their benefits are well established but few are ongoing researches that if gets success will definitely add up a lot to their importance in our diet. Therefore, our further studies will emphasis on researching and carrying out experiments that can be applied on human beings and give fruitful results. The anti-cancer property of papaya leaf, blood platelet count increase in dengue affected patients by consuming papaya leaf extract or juice. These experiments needs proper case study to find its authentication. This is our scope of further study

\section{Acknowledgement}

We would like to thank our project guide, our laboratory teachers, respective faculty members for constant support and encouraging us for this project. Sincere regards to all scientists, researchers, and eminent persons from the academic as well as research field whose journals, publications provided us with the constant support and also our college for helping us financially

\section{REFERENCES}

[1] Priyanka Priyanka, Dileep Kumar, Anurag Yadav, Kusum Yadav, U. N. Dwivedi, "Analysis of Simple Sequence Repeats Information from Floral Expressed Sequence Tags Resources of Papaya (Carica papaya L.)", Vol.8 No.9, American Journal of Plant Sciences, August 24, 2017.

[2] Storey WB, Evolution of Crop Plants", NW Edition, Longman Publications, London, UK, 1976.

[3] Ock Kyoung Chun Dae-Ok Kim Nancy Smith David Schroeder Jae Taek Han Chang Yong Lee, "Daily consumption of phenolics and total antioxidant capacity from fruit and vegetables in the American diet", Volume 85 , Number 10, Journal of the Science of Food and Agriculture, John Wiley \& Sons, Ltd., 15 August 2005.

[4] Nor Azrina Norahmad, Mohd Ridzuan Mohd, Abd Razak, Norazlan Mohmad Misnan, Nur Hana Md Jelas, Umi Rubiah Sastu, Amirrudin Muhammad, Tiffiny Chau Dee Ho, Bazilah Jusoh, Nor Azlina Zolkifli, Ravindran Thayan, Adiratna Mat Ripen, Murizal Zainol and Ami Fazlin Syed Mohamed, "Effect of freeze-dried Carica papaya leaf juice on inflammatory cytokines production during dengue virus infection in AG129 mice", Volume 19, Article Number 44, BMC Complementary Medicine and Therapies, $11^{\text {th }}$ February, 2019.

[5] Sengupta, Susmita \& Sengupta, L \& Bisen, Prakash, "Bioengineered Crops: The Commercial and Ethical Considerations", Volume 2, Current Genomics, 2001.

[6] Zuhrotun Nisa F, Astuti M, Murdiati A, Mubarika Haryana $\mathrm{S}$, "Anti-proliferation and Apoptosis Induction of Aqueous Leaf Extract of Carica papaya L. on Human Breast Cancer Cells MCF-7", Volume 20, Pakistan Journal of Biological Sciences, 2017.

[7] Rajesh Verma, Pawan Sharma, Ravindra Kumar Garg, Veerendra Atam, Maneesh Kumar Singh, Hardeep Singh Mehrotra, "Neurological complications of dengue fever: Experience from a tertiary center of north India", Volume 14, Annals of Indian Academy of Neurology, 2011.

[8] Saurabh Pandey, Peter J. Cabot, P. Nicholas Shaw and Amitha K. Hewavitharana, "Anti-inflammatory and immunomodulatory properties of Carica papaya", Volume 13, Journal of Immunotoxicology, 14th July 2016.

[9] Q. V. Vuong, S. Hirun, P. D. Roach, M. C. Bowyer, P. A. Phillips, and C. J. Scarlett, "Effect of extraction condition on total phenolic compounds and antioxidant activities of Carica papaya leaf aqueous extract," Volume 3, Journal of Herbal Medicine, 2013.

[10] Sharifah Nuruljannah Syed Amran, Noraziani Zainal Abidin, Haslaniza Hashim, and Saiful Irwan Zubairi, "Saponin Bitterness Reduction of Carica papaya Leaf Extracts through Adsorption of Weakly Basic Ion Exchange Resins", Volume 2018, Journal of Food Quality, 24th September, 2018.

[11] Kulkarni, A.P., S.M. Aretha and S. Divakar, "Isolation and identification of a radical scavenging antioxidant punicalagin from pith and carpellary membrane of pomegranate fruit”, Volume 87, October, 2004.

[12] R. P. Singh, K. N. Chidambara Murthy and G. K. Jayaprakasha, "Studies on the Antioxidant Activity of Pomegranate (Punicagranatum) Peel and Seed Extracts Using in Vitro Models", Volume 50, Journal of Agricultural and Food Chemistry, 17th November, 2001.

[13] Eirini Manthou, Kalliopi Georgakouli, Chariklia K. Deli, Aggelos Sotiropoulos, Ioannis G. Fatouros, Dimitrios Kouretas, Serko
Haroutounian, Chrysoula 
Matthaiou, Yiannis Koutedakis and Athanasios Z. Jamurtas, "Effect of pomegranate juice consumption on biochemical parameters and complete blood count", Volume 14, Spandidos Publications, 27 th June, 2017.

[14] Ock Kyoung Chun, Dae-Ok Kim ,Nancy Smith, David Schroeder, Jae Taek Han and Chang Yong Lee, "Daily consumption of phenolics and total antioxidant capacity from fruit and vegetables in the American diet", Volume 85, Journal of the Science of Food and Agriculture, $31^{\text {st }}$ March, 2005.

[15] Guihua Xu, Donghong Liu, Jianchu Chen, Xingqian Ye, Yaqin Ma and John Shi, "Juice components and antioxidant capacity of citrus varieties cultivated in China", Volume 106, Journal of Food Chemistry, Elsevier, $5^{\text {th }}$ June, 2007.

[16] Beatriz Álvarez Arias and LuisRamón-Laca, "Pharmacological properties of citrus and their ancient and medieval uses in the Mediterranean region", Volume 97, Journal of Ethnopharmacology, 10 ${ }^{\text {th }}$ February, 2005.

[17] BK Srikanth, Lakshminarayana Reddy, Sunita Biradar, Manjula Shamanna, Deepak D Mariguddi, M Krishnakumar, "An open-label, randomized prospective study to evaluate the efficacy and safety of Carica papaya leaf extract for thrombocytopenia associated with dengue fever in pediatric subjects, Volume 10 of 2019, Professor Roosy Aulakh, 17 th January, 2019

[18] Senanayake A M Kularatne, "Dengue Fever", BMJ (United Kingdom), 15 $5^{\text {th }}$ September, 2015.

[19] Hettige S., "Salutary effects of Carica papaya leaf extract in dengue fever patients- a pilot study", Volume 29, BMJ (United Kingdom), 2008.

[20] Thao T. Nguyen, Marie-Odile Parat, Mark P. Hodson, Jenny Pan,Paul N. Shaw and Amitha K. Hewavitharana, "Chemical Characterization and in Vitro Cytotoxicity on Squamous Cell Carcinoma Cells of Carica Papaya Leaf Extracts", Volume 8, MDPI Open Access Journals, 24th December, 2015

[21] Fatin Najwa and Azrina Azwal, "Comparison of vitamin C content in citrus fruits by titration and high performance liquid chromatography (HPLC) methods", Volume 2, International Food Research Journal, April, 2017.

[22] Sadiye Gozlekci, Onur Saracoglu, Ebru Onrusal and Mustafa Ozgen, "Total phenolic distribution of juice, peel and seed extracts of four pomegranate cultivars", Volume 7, Pharmacognosy Magazine, 9 ${ }^{\text {th }}$ May, 2011

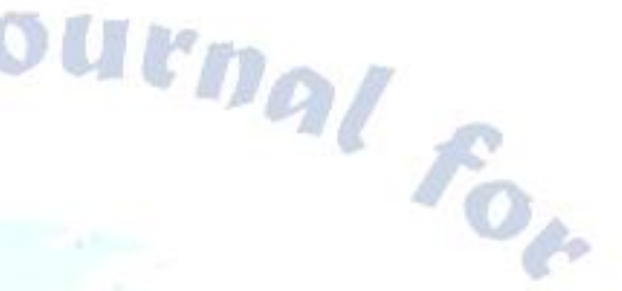

